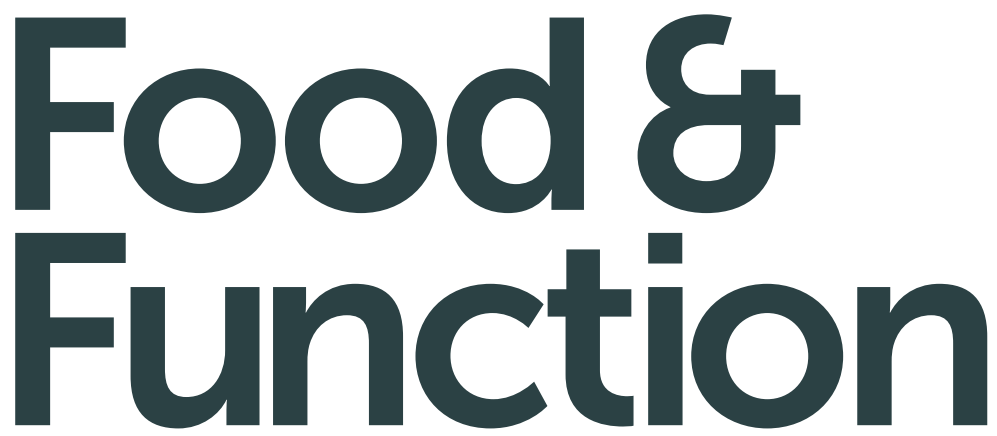

Linking the chemistry and physics of food with health and nutrition rsc.li/food-function

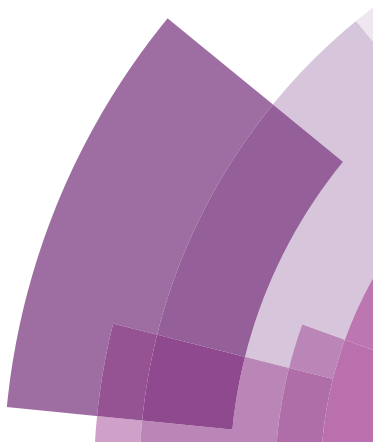




\section{A Check for updates}

Cite this: Food Funct., 2019, 10, 7645

\title{
A randomized, double-blind, placebo-controlled pilot study to assess bacterial anti-adhesive activity in human urine following consumption of a cranberry supplement $\dagger$
}

\begin{abstract}
Haiyan Liu, amy B. Howell, (DD ${ }^{b}$ Derek J. Zhang ${ }^{a}$ and Christina Khoo (D) *a
Urinary tract infections (UTIS) are one of the common bacterial infections treated with antibiotics. The North American cranberry is recommended for prophylaxis in women with recurrent UTIs as a nutritional alternative. The ability of cranberry components and their metabolites to inhibit adhesion of uropathogenic Escherichia coli (E. coli) is an important mechanism by which cranberry mitigates UTIs. The objective of this study was to evaluate urinary anti-adhesion activity against type 1 and P-type uropathogenic E. coli after consumption of cranberry +health ${ }^{\mathrm{TM}}$ cranberry supplement (cranberry chew). In this randomized, double-blind, placebo-controlled, crossover design pilot trial $(n=20)$, subjects consumed two cranberry or placebo chews, one in the morning and one in the evening. Clean-catch urine samples collected at the baseline and post-intervention $(0-3,3-6,6-9,9-12,12-24,24-30,30-36$ h) were tested for anti-adhesion effects with a mannose-resistant human red blood cell hemagglutination assay specific for P-type E. coli, or a T24 cell line model for type 1 E. coli. Urinary anti-adhesion activity against P-type E. coli after consumption of the cranberry chew was significantly greater $(p<0.05)$ than that observed with placebo chew at all time points except 24-36 h. Ex vivo anti-adhesion effects on type $1 E$. coli were greater $(p<0.05)$ after cranberry chew consumption than placebo chew at 3-6 and 6-9 h urine collections. In conclusion, consumption of cranberry +health ${ }^{\mathrm{TM}}$ cranberry supplement exhibited greater ex vivo urinary anti-adhesion activity compared to placebo, suggesting that it may have the potential to help promote urinary tract health.
\end{abstract}

Received 5th June 2019,

Accepted 9th September 2019

DOI: 10.1039/c9fo01198f

rsc.li/food-function health have been recommended for women with recurrent UTIs. $^{4-6}$ Two recent meta-analyses supported the beneficial effects of cranberry consumption on reducing risk of UTIs. Fu et al. found that cranberry reduced the risk of UTIs by $26 \%$ (pooled risk ratio: $0.74 ; 95 \% \mathrm{CI}: 0.55,0.98 ; I^{2}=54 \%$ ), ${ }^{4}$ and Luis et al. showed that cranberry products significantly reduced the incidence of the infections with a weighted risk ratio of 0.675 (95\% CI $0.5516-0.7965, p<0.0001)$ for a heterogeneous population. ${ }^{5}$ However, inconsistent evidence is still being observed in some clinical trials ${ }^{7}$ and may be attributed to various factors including inappropriate subject population, high withdrawal rate or poor compliance, lack of standardization of cranberry bioactive components in administered treatment products and suboptimal dosing.

Uropathogenic Escherichia coli (UPEC) is the most common pathogen causing 75-95\% of uncomplicated cystitis and pyelonephritis. ${ }^{8}$ UPEC expresses a broad spectrum of virulence factors with pathogenicity being determined predominantly by surface adhesive molecules. To initiate an infection, uropathogens first utilize their fimbriae or pili to adhere to the uroepithelial cell receptors, leading to bacterial proliferation. ${ }^{9}$ 
Three UPEC fimbrial types are associated with most infections. UPEC expressing type 1 fimbriae primarily bind to uroplankin Ia receptors causing the majority of acute cystitis. ${ }^{10}$ P-fimbriated UPEC plays an important role in the pathogenesis of ascending UTIs and pyelonephritis ${ }^{11}$ and UPEC expressing F9 fimbriae are associated with binding to chronic and inflamed bladder epithelium. ${ }^{12}$ Extensive research has been conducted to investigate bioactive components in cranberries that are responsible for the anti-adhesion activity. Previous studies have shown that cranberry polyphenolic compounds such as proanthocyanidins, ${ }^{13,14}$ flavonols and their glycosides (e.g. myricetin, quercetin) ${ }^{15,16}$ condensed tannins (e.g. procyanidins, prodelphinidin) ${ }^{15,17}$ and phenolic acids (e.g. benzoic acid $)^{18}$ were able to inhibit the in vitro adherence of P-type E. coli to the glycoprotein receptor on epithelial cells or red blood cell analogues. Certain parent phenolic components in the cranberries and gut microbial derived phenolic catabolites (e.g. phenols, valerolactone and phenolic acids) showed antiadhesion potential. Two of the most abundant metabolites (hippuric acid and $\alpha$-hippuric acid) after cranberry consumption exhibited anti-adhesion effects on P-type UPEC at physiologically relevant concentrations $(100$ to $500 \mu \mathrm{M}) \cdot{ }^{18}$ In addition, metabolites such as catechol, vanillic acid, phenylacetic acid and 3,4-dihydroxyphenylacetic acid were also able to inhibit the adherence of P-type UPEC to uroepithelia cells in a concentration-dependent manner. ${ }^{18}$ Moreover, the gut microbial degradation products of cranberry PACs, valerolactone and its derivatives exhibited anti-adhesion properties against P-type UPEC at a concentration of $100 \mu \mathrm{M}$, indicating their potential biologically relevant activity in urine. ${ }^{19}$ The anti-adhesion activities of cranberry-derived metabolites suggest that their presence in the urine could be responsible for the further reduction of bacterial colonization and pathogenesis of UTIs.

Most ex vivo studies using urine from subjects after cranberry consumption have been focused on anti-adhesion effects on UPEC expressing P-fimbriae. ${ }^{20-23}$ Only a few studies have investigated the inhibitory effects of cranberry consumption on UPEC expressing type 1 fimbriae. ${ }^{24,25}$ The objective of this study was to investigate the urinary anti-adhesion activity against both type 1 and P-fimbriae UPEC in healthy individuals following the consumption of cranberry products in a pilot trial. We performed this randomized, double-blind, placebo-controlled, crossover design pilot trial to investigate whether the cranberry chew (cranberry thealth ${ }^{\mathrm{TM}}$ cranberry supplement) would provide strong ex vivo anti-adhesion effects in the urine compared to a placebo chew.

\section{Experimental}

\subsection{Clinical study design}

The pilot study was approved by the Institutional Review Board at Rutgers University. Twenty healthy male and female subjects without a history of current or recurrent urinary tract infections, urinary disorders, or antibiotic use within the last six months were recruited for this randomized, double-blind, placebo-controlled, crossover design pilot trial. All subjects were provided a list of foods to avoid during the 7-day run-in period prior to the intervention and throughout the duration of the study. They include Vaccinium fruits and high polyphenolic foods including cranberries, blueberries, lingonberries, chocolate, red wine, strawberries, grapes, cherries, plums, blackberries, etc. On the morning of the intervention between 7:00 and 10:00 AM, a baseline urine sample $(t=0 \mathrm{~h})$ was collected from all subjects after overnight fasting. Participants were then randomized to receive either 1 cranberry chew or 1 placebo chew with $240 \mathrm{~mL}$ water. The cranberry chew contained cranberry fruit extract, apple juice concentrate, apple puree and pectin. The placebo chew was made from apple juice concentrate, apple puree and pectin and formulated to match the texture and appearance of the active chew without cranberry. Each type of chew was randomly assigned a 3-digital code generated by a computer system. Both volunteers and investigators were blinded to the treatment. Clean-catch urine samples were collected at 0-3, 3-6, 6-9 and 9-12 h post-consumption. Multiple urine collections made within each time interval were pooled. In the same day between 7:00 and 10:00 PM $(t=12 \mathrm{~h})$, participants consumed another 1 cranberry chew or 1 placebo chew with $240 \mathrm{~mL}$ water. The following morning, an overnight urine sample $(t=12-24 \mathrm{~h})$ was self-collected and submitted to the clinic lab. In the afternoon and evening, urine samples were also collected at $t=24-30 \mathrm{~h}$ and $t$ $=30-36 \mathrm{~h}$. After a one-week wash out, participants then began the alternative regimen and repeated the protocol. All urine samples $(0,0-3,3-6,6-9,9-12,12-24,24-30,30-36 \mathrm{~h})$ were aliquoted and kept in a $-80{ }^{\circ} \mathrm{C}$ freezer until analysis. All experiments were performed in accordance with the Guidelines: Policy for Human Subjects Protection and the Institutional Review Board. Experiments were approved by the ethics committee at the Office of Research and Regulatory Affairs, Rutgers University. Informed consent was obtained from human participants of this study.

\subsection{Mannose-resistant hemagglutination (MRHA) assay (P-type E. coli)}

Anti-adhesion activity against P-fimbriae E. coli was tested using a MRHA assay. The ability of urine samples to suppress agglutination of mannose-resistant human red blood cells (HRBC; A1, Rh+) with a clinical $E$. coli isolate was evaluated according to protocols as previously described. ${ }^{21,22}$ Results were reported on an ordinal scale with anti-adhesion activity as follows: $0=0 \%$ adhesion inhibition; $1=50 \%$ inhibition; and $2=100 \%$ inhibition. Negative controls included wells containing bacteria + PBS, HRBC + PBS, bacteria + urine, and HRBC + urine. A well containing bacteria + HRBC served as a positive control for agglutination.

\subsection{Type 1 fimbriated $E$. coli-uroepithelial cell anti-adhesion assay}

Gram-negative E. coli strain UTI89 was originally isolated from a woman with acute cystitis. ${ }^{26}$ Anti-adhesion activity against type 1 fimbriated $E$. coli was measured by first incubating 
BacLight Green labeled bacteria with urine samples or with myricetin in a 96-well microplate for $30 \mathrm{~min}$ at $36{ }^{\circ} \mathrm{C}$. Myricetin was applied as a positive control due to its known bacterial anti-adhesion capability. ${ }^{15,27,28}$ In our previous collaborative research, myricetin dose-dependently inhibited $E$. coli adherence with a half-maximal inhibitory concentration $\left(\mathrm{IC}_{50}\right)$ of $0.88 \mu \mathrm{g} \mathrm{mL}{ }^{-1} \cdot{ }^{15}$ Briefly, $200 \mu \mathrm{L}$ of treated bacteria were transferred to the solid black 96-well microplate containing fixed uroepithelial cells, followed by centrifugation at $500 \mathrm{~g}$ for $2 \mathrm{~min}$. The plate was incubated at $37{ }^{\circ} \mathrm{C}$ under a $5 \% \mathrm{CO}_{2}$ atmosphere for $1 \mathrm{~h}$. To remove the excess unbound bacteria, the supernatant was removed and each well was washed once with DPBS without $\mathrm{Ca}^{2+} / \mathrm{Mg}^{2+}$. Fluorescence intensity (FI) was acquired in a microplate reader and quantified with an excitation wavelength of $485 \mathrm{~nm}$ and an emission wavelength of $535 \mathrm{~nm}$. All urine samples and the positive control compound were tested in triplicate and standardized to creatinine in the urine.

\subsection{Type 1 anti-adhesion activity and anti-adhesion potency determination}

A standard inhibition curve was generated by incubating BacLight labeled bacteria with myricetin at different concentrations $\left(0,0.125,0.25,0.5,1.25,2.5\right.$ and $\left.5 \mu \mathrm{g} \mathrm{mL}{ }^{-1}\right)$ at $35{ }^{\circ} \mathrm{C}$ for $30 \mathrm{~min}$. A linear regression equation was generated based on the fluorescence intensity (FI) of standards. Myricetin equivalency in each sample was projected from the regression equation based on FI in individual samples. Results were expressed as $\mu \mathrm{g}$ myricetin per $\mathrm{mL}$. The final anti-adhesion activity was adjusted by dividing the myricetin equivalency in each urine sample by its respective creatinine content, expressed as $\mu \mathrm{g}$ myricetin per mg of creatinine.

The anti-adhesion potency of individual urine samples was expressed as \% of anti-adhesion activity with respect to the $\mathrm{IC}_{50}$ of all myricetin standard inhibition curves recorded. The mean $\mathrm{IC}_{50}$ of the myricetin standard curve is 1.037 .

$$
\begin{aligned}
& \text { Anti - adhesion potency }= \\
& \qquad \frac{\text { anti }- \text { adhesion activity }}{\text { average } \text { IC }_{50} \text { of myricetin standard curve }}
\end{aligned}
$$

\subsection{Analytical measurement of bioactive components in chews}

The total proanthocyanidin (PAC) content was determined in a 4-dimethylaminocinnamaldehyde (DMAC) colorimetric assay with an absorbance at $640 \mathrm{~nm}$ wavelength. The assay utilized either a procyanidin A2 dimer as a reference standard (BL-DMAC method, adapted from the study by Prior et al.) or a proprietary reference standard based on an isolated fraction of purified cranberry PAC developed by Ocean Spray Cranberries, Inc. (OSC-DMAC method). ${ }^{29,30}$ The standard used in the OSC-DMAC method would represent more accurately of spectrum of degree of polymerization (DP) of PAC in the cranberries, which would expect to yield a higher value of PAC in comparison with the BL-DMAC method. The DP profile was quantified by using an Agilent 1100/1200 HPLC system with fluorescence detection as described previously. ${ }^{31}$ The column was a Develosil Diol 100A $(250 \times 4.6 \mathrm{~mm}, 5 \mu \mathrm{m}$ particle size $)$ with a flow rate of $1 \mathrm{~mL} \mathrm{~min}^{-1}$. The mobile phase was a binary gradient consisting of acidic acetonitrile (solvent A, acetonitrile: acetic acid, $98: 2, \mathrm{v}: \mathrm{v}$ ) and acidic aqueous methanol (solvent $\mathrm{B}$, methanol : water : acetic acid, $95: 3: 2, \mathrm{v}: \mathrm{v}: \mathrm{v})$. The starting mobile phase condition is $7 \% \mathrm{~B}$; hold isocratic for $3 \mathrm{~min}$. Subsequently, solvent B was ramped to $37.6 \%$ for 57 min and to $100 \% \mathrm{~B}$ for $3 \mathrm{~min}$ thereafter. The condition was held at $100 \%$ B for 7 min prior to returning to the starting condition. Fluorescence detection was determined with an excitation and emission wavelength of 230 and $321 \mathrm{~nm}$, respectively. Data obtained from the BL-DMAC method should be interpreted as milligrams (mg) of procyanidin A-2 equivalents per gram (g) of powder or millilitre $(\mathrm{mL})$ of juice. The data generated using the Ocean Spray method (OSC-DMAC) to measure PACs should be interpreted as milligrams $(\mathrm{mg})$ of cranberry specific PAC equivalents per gram $(\mathrm{g})$ of powder or millilitre $(\mathrm{mL})$ of juice.

Total phenolics was measured using an improved FolinCiocalteu method and absorbance was measured at $765 \mathrm{~nm}$ wavelength. ${ }^{32}$

Anthocyanins were analyzed according to Brown and Shipley $^{33}$ using a HPLC system (Agilent 1260 Infinity, Agilent Technologies, Inc., Santa Clara, CA, USA) equipped with a binary pump, an autosampler and a diode array detector (DAD). The mobile phase consisted of solvent A (deionized water : 85\% phosphoric acid, $99: 1, \mathrm{v}: \mathrm{v}$ ) and solvent B (deionized water: acetonitrile : $85 \%$ phosphoric acid, $50: 49: 1, \mathrm{v}: \mathrm{v})$. The gradient was as follows: 0-1 min, 0-10\% B linear; 1-28 min, 10-50\% B linear; 28-32 $\mathrm{min}, 50-75 \% \mathrm{~B}$ linear; and 32-32.1 min, 75-10\% B linear; 32.1-35 min, 10\% B isocratic. The column temperature was set at $25^{\circ} \mathrm{C}$ and data were collected at $520 \mathrm{~nm}$ (DAD). Peaks were compared to those of commercially available cyanidin-3galactoside, cyanidin-3-glucoside, and peonidin-3-glucoside standards (ChromaDex, Irvine, CA USA).

Flavonoid analyses were performed on an Agilent 1260 HPLC system (Agilent 1260 Infinity, Agilent Technologies, Inc., Santa Clara, CA, USA) equipped with a binary pump, an autosampler and a DAD. Chromatographic separation was carried out with a Phenomenex Kinetex $2.6 \mu$ F5, $100 \AA 100 \times 4.6 \mathrm{~mm}$ column (Torrance, CA, USA). The mobile phase consisted of solvent A (deionized water : trifluoroacetic acid, 99.9: 0.1, v:v) and solvent B (acetonitrile : methanol, $70: 30, \mathrm{v}: \mathrm{v}$ ). The gradient was as follows: 0-2 $\mathrm{min}, 14 \% \mathrm{~B}$ isocratic; 2-9 $\mathrm{min}, 14-18 \%$ B linear; 9-22 min, 18-43\% B linear; 22-23 min, 43-90\% B linear; $23-24 \mathrm{~min} 90 \% \mathrm{~B}$ isocratic, and $24-25 \mathrm{~min}, 90-14 \% \mathrm{~B}$ linear. The column temperature was set at $30{ }^{\circ} \mathrm{C}$ and data were collected at 360, 320 and $280 \mathrm{~nm}$ (DAD). Peaks were compared to those of commercially available standards.

\subsection{Statistical analyses}

Non-parametric analyses were conducted in a Minitab 18 (Minitab, Inc., State College, PA, USA). For type-P anti-adhesion assay, the difference in anti-adhesion activity between the cranberry chew and placebo chew at each time point was evaluated by the Wilcoxon signed rank test. Due to the highly skewed dataset in type-1 anti-adhesion assay, the Mann-Whitney test was assigned to determine the significant treatment effect 
between the cranberry chew and placebo chew. A $p$-value of less than 0.05 was considered statistically significant.

\section{Results}

\subsection{Analytical characterization of the cranberry chew}

One $9 \mathrm{~g}$ serving of the cranberry chew has a caloric content of $25 \mathrm{kcal}$ and $6 \mathrm{~g}$ of sugar with levels of important phenolic con-

Table 1 Analytical characterization of cranberry chew

\begin{tabular}{lll}
\hline & $\begin{array}{l}\text { Cranberry } \\
\text { chew }\end{array}$ & $\begin{array}{l}\text { Placebo } \\
\text { chew }\end{array}$ \\
\hline Serving size (g) & 9 & 9 \\
Calories (kcal) & 25 & 25 \\
Total carbs/sugar (g) & $7 / 6$ & $7 / 6$ \\
Cranberry fruit extract & YES & NO \\
Vitamin C (mg) & ND & ND \\
Proanthocyanidins by OSC-DMAC (mg) & $120.14 \pm 17.27$ & ND \\
Proanthocyanidins by BL-DMAC (mg) & $29.35 \pm 6.08$ & ND \\
Total phenolics by Folin-Ciocalteu & $162.09 \pm 4.62$ & $14.00 \pm 0.85$ \\
(mg) & & \\
Total phenolic acid content (mg) & $8.47 \pm 0.49$ & ND \\
Total organic acid content (mg) & $530.06 \pm 9.07$ & $280.63 \pm 79.17$ \\
Cyanidin-3-arabinoside (mg) & $0.40 \pm 0.17$ & $0.01 \pm 0.00$ \\
Cyanidin-3-galatoside (mg) & $0.78 \pm 0.35$ & $0.03 \pm 0.00$ \\
Cyanidin-3-glucoside (mg) & $0.03 \pm 0.01$ & ND \\
Peonidin-3-arabinoside (mg) & $0.42 \pm 0.18$ & $0.01 \pm 0.00$ \\
Peonidin-3-galatoside (mg) & $0.99 \pm 0.46$ & $0.05 \pm 0.00$ \\
Peonidin-3-glucoside (mg) & $0.10 \pm 0.04$ & ND \\
Total anthocyanin content (mg) & $2.72 \pm 1.21$ & $0.76 \pm 0.03$ \\
Myricetin (mg) & $0.61 \pm 0.11$ & ND \\
Myricetin-3-O-galactoside (mg) & $2.27 \pm 0.20$ & ND \\
Myricetin-3-O-rhamnoside (mg) & $0.58 \pm 0.16$ & ND \\
Quercetin (mg) & $1.32 \pm 0.11$ & ND \\
Quercetin-3-O-galactoside (mg) & $5.03 \pm 0.22$ & ND \\
Quercetin-3-O-rhamnoside (mg) & $1.17 \pm 0.11$ & ND \\
Unidentified flavonols (mg) & $2.02 \pm 0.44$ & ND \\
Total flavonol content (mg) & $13.00 \pm 1.35$ & ND \\
& & \\
& &
\end{tabular}

Data are expressed as mean \pm standard deviation. ND: not detected. stituents (Table 1) similar to one $8 \mathrm{oz}$ serving of a cranberry beverage tested in the large clinical trial in women with recurrent UTIs. ${ }^{6}$ The PAC content in the cranberry chew was 24.39-36.13 mg A2 equivalents per serving using BL-DMAC assay, and 103.37-137.87 mg per serving by the OSC-DMAC method (Table 1), which is higher than that in most other cranberry supplements on the market (unpublished internal data). The degree of polymerization (DP) of cranberry PACs in chew was quantified. Monomers (DP1), dimers (DP2), trimers (DP3), oligomers (DP4-7), and polymers (DP $\geq 10$ ) accounted for $1.6 \%, 4.2 \%, 3.8 \%, 16.3 \%$, and $74 \%$, respectively, of total cranberry PACs (120 mg per $9 \mathrm{~g}$ chew by OSC-DMAC method). The characteristic anthocyanin and flavonol profiles of cranberry fruits were detected in cranberry chew as shown in Table 1 . The total phenolic content in cranberry chew was $162.1 \mathrm{mg}$ gallic acid equivalents per serving.

\subsection{Urinary anti-adhesion activity against P-fimbriated $E$. coli}

Urinary anti-adhesion activity against P-type E. coli was evaluated using MRHA assay and the results are shown in Fig. 1. No anti-adhesion activity was reported in $0 \mathrm{~h}$ baseline urine samples taken prior to the consumption of treatment products. Significantly greater $(p<0.05)$ anti-adhesion activity was observed in urine samples collected from volunteers that consumed cranberry chew compared to placebo chew at all collection time points except for $24-30 \mathrm{~h}$ and $30-36 \mathrm{~h}$. The activity peaked between 3 and $6 \mathrm{~h}$ following the consumption of the first dose of cranberry chew and lasted up to $12 \mathrm{~h}$. These results are consistent with our previous study in which urinary anti-adhesion activity was significantly greater compared to placebo after consumption of cranberry extract beverage/cranberry extract and juice beverage, with activity peaking between 3 and $6 \mathrm{~h}$, but lasting only $9 \mathrm{~h}^{22}$ To build on the learning from the previous study, volunteers were given a second dose cranberry chew to consume at $t=12 \mathrm{~h}$, with additional urine collections. Urine collected in the morning after the second

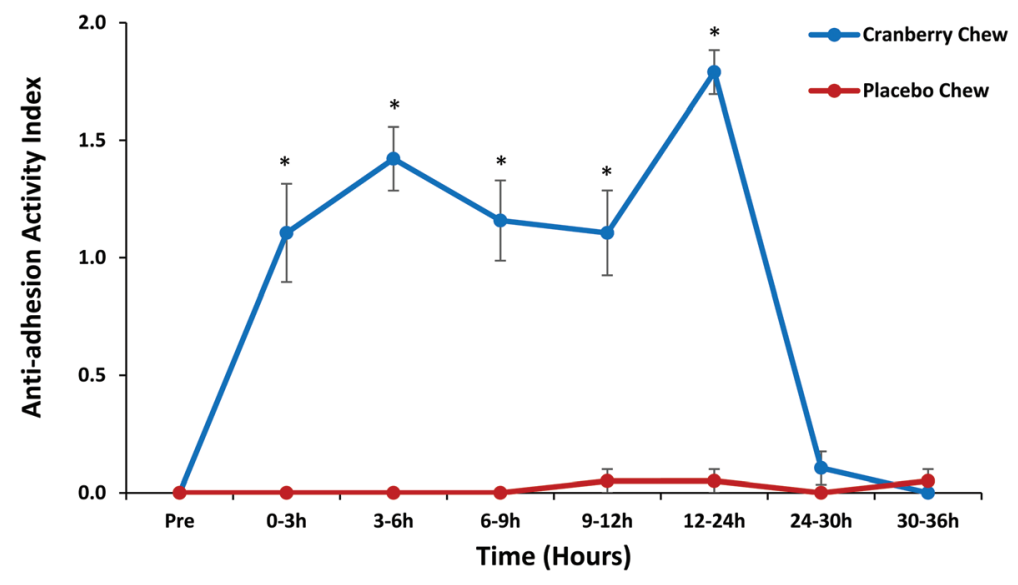

Fig. 1 Urinary anti-adhesion activity against $\mathrm{P}$ fimbriated uropathogenic $E$. coli after consumption of two divided doses of cranberry chew. Data are expressed as mean \pm SEM $(n=20)$. Anti-adhesion index key: $1=50 \%$ anti-adhesion activity, $1.5=75 \%$ anti-adhesion activity and $2.0=100 \%$ antiadhesion activity. * indicates that post-consumption anti-adhesion activity of cranberry chew was significantly greater compared to that of placebo chew $(p<0.05)$. 
dose showed the highest anti-adhesion activity (AAA $=1.8$, Fig. 1) over the time course of the study, indicating the possibility of extending the benefit to the urinary tract from the second dose of cranberry chew. Anti-adhesion activity decreased to near baseline values at $24-30 \mathrm{~h}$ and $30-36 \mathrm{~h}$ after consumption of the first dose. No anti-adhesion activity was reported for placebo chew at any time.

\subsection{Urinary anti-adhesion activity against type $1 \mathrm{E}$. coli}

In the present study, we successfully optimized and validated the published fluorometric microplate assay by Kimble et al. ${ }^{15}$ and demonstrated the urinary anti-adhesion effects on type 1 E. coli following cranberry chew consumption. The data (Fig. S1A and $\mathrm{S} 1 \mathrm{~B} \dagger$ ) showed that a BacLight Green concentration of $1000 \mu \mathrm{M}$ and a ratio of UPEC : uroepithelial cells of $600: 1$ were optimal and physiologically relevant.

Urine samples collected from volunteers who consumed cranberry or placebo chews were analyzed using the current fluorescent-labeled UPEC and uroepithelial cell assay format to evaluate anti-adhesion effects on type $1 \mathrm{E}$. coli. Urine samples collected from four subjects were not tested due to anti-adhesion activity being detected in their pre-study background urine samples. This phenomenon could be due to the production of endogenous adhesion inhibitors such as Tamm-Horsfall glycoprotein. ${ }^{34,35}$ Urine samples from a total of 16 subjects were analyzed further for their type 1 antiadhesion effect (Fig. 2). Urine samples other than those collected at 24-30 $\mathrm{h}$ and 30-36 h exhibited some anti-adhesion activity on type $1 \mathrm{E}$. coli following cranberry chew consumption and the results obtained were consistent with results obtained in the MRHA assay using P-fimbriae E. coli. The type 1 antiadhesion activity peaked at 3-6 h $(p=0.024)$ after the first chew dose and lasted up to 9 hours $(p=0.043)$ when the effect was both significantly different from placebo chew. The activity decreased in urine collected from 9-12 $\mathrm{h}$ and peaked again in the $12-24 \mathrm{~h}(p=0.12)$ urine samples after consuming a second dose cranberry chew (Fig. 2).

\section{Discussion}

Cranberries are known to be a rich source of phenolic compounds including polymeric flavan-3-ols (PACs), anthocyanins and flavonols. High levels of phenolic constituents were detected in cranberry chew, with PAC levels (Table 1) greater than many other commercial cranberry supplements (unpublished internal data). The levels of phenolic content were similar to those in a cranberry beverage recently found in a clinical trial to reduce symptomatic UTI by $39 \% .{ }^{6}$ Cranberry PACs are unique in the structure with a higher percentage of A-type bonds, compared with other commonly consumed fruits. In a previous study conducted by Feliciano and colleagues, ${ }^{36}$ cranberry PACs with one A-type bond were found most abundant in DP between 2 and 5. PACs with two A-type bonds were most abundant in DP between 6 and 9. PACs with DP 10 and 11 exhibited mainly three A-type bonds. In another study performed by the same research group, ${ }^{37}$ results showed that $94.5 \%$ of cranberry PACs contained at least one A-type bond and 26\% PACs had at least two A-type bonds. This was in contrast to the DP profile of apple PACs with $88.3 \%$ as B-type bonds. Compared with apple PACs, cranberry A-type PACs were significantly more effective in increasing bacteria agglutination and decreasing bacteria invasion to epithelial cells caused by extra-intestinal pathogenic E. coli. ${ }^{37}$ The result was consistent with a previous finding that the urinary tract benefit was only observed following cranberry intervention but not after the use of B-type PAC rich food, such as grape, apple juice, green tea, and dark chocolate. ${ }^{13}$ It is noted that oligomers and polymers of PACs are flavan-3-ol with a high molecular weight and were minimally absorbed in the upper GI tract ${ }^{38}$ until they reached

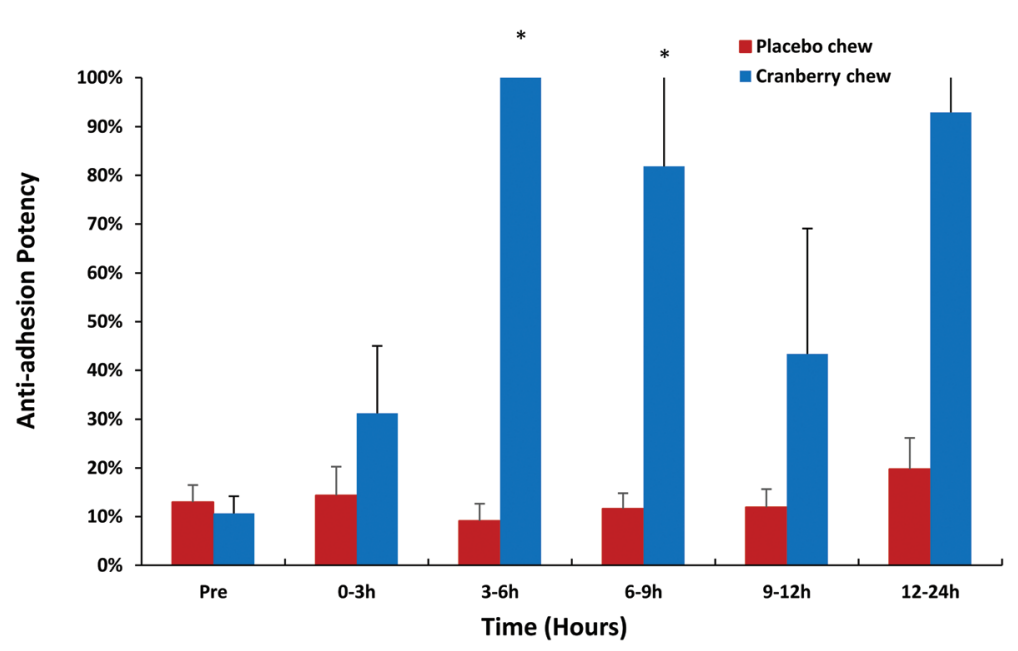

Fig. 2 Urinary anti-adhesion activity against type 1 fimbriated uropathogenic $E$. coli after consumption of two divided doses of cranberry chew. Data are expressed as mean \pm SEM $(n=16)$. The anti-adhesion potency of individual urine samples was expressed as \% of anti-adhesion activity with respect to the mean half-maximal inhibitory concentration $\left(E_{50}\right)$ of all myricetin standard curves recorded. The mean $\mathrm{EC}_{50}$ of the myricetin standard curve is 1.037. * indicates that post-consumption anti-adhesion activity of cranberry chew was greater compared to that of placebo chew $(p<0.05)$. 
colon for fermentation. It is likely that the bacterial metabolites from PACs that were absorbed and excreted in the urine elicit the benefit. Ou et al. showed that cranberry procyanidins did not yield the exclusive metabolite profile after gut bacteria fermentation, compared with apple procyanidins but had a slower degradation rate by gut bacteria, yielding higher levels of certain bacteria-derived metabolites compared to the apple procyanidins. ${ }^{39}$ This suggested that bacteria derived enzyme(s) might interact and utilize A-type and B-type PACs differently due to the difference in the configuration of A-type and B-type PACs.

Anthocyanins make up another important class of polyphenols in cranberries, providing the red color of the berries and eliciting certain bioactivities, such as antioxidant and antiinflammatory activities. ${ }^{40}$ The levels were equivalent to those found in raw cranberry fruits, suggesting that most of the anthocyanins are retained in the cranberry chew following the manufacturing process. It has been reported that more than 20 different glycosides of flavonols ${ }^{41-43}$ are found in cranberries and the contents are nearly double those found in commonly consumed fruits including pomegranates and grapes. ${ }^{44,45}$ The most abundant flavonols in cranberries are quercetin-3-galactoside and myricetin-3-O-galactoside which were retained in the cranberry chew at levels similar to fresh cranberries $(29.68 \mathrm{mg}$ quercetin-3-galactoside $14.8 \mathrm{mg}$ myricetin-3-O-galactoside per $100 \mathrm{~g}$ fruit) (Table 1). Quercetin and quercetin-3-rhamnoside (Table 1) were much higher compared to those in cranberry fruits $(0.67 \mathrm{mg}$ quercetin and $2.65 \mathrm{mg}$ quercetin-3-rhamnoside per $100 \mathrm{~g}$ fruit). ${ }^{9}$ The cranberry chew also contained a high level of various phenolic acids, such as benzoic acid, chlorogenic acid, 3,4-hydroxylbenzoic acid, and p-coumaric acid (Table 1), which may possess high anti-microbial properties. ${ }^{28}$

In vitro studies have shown cranberries and certain polyphenolic compounds to possess some anti-adhesion activity when directly incubated with bacteria and cultured cells. ${ }^{13-18}$ However, it is difficult to determine if these compounds will be active in vivo and at what concentrations. Rodríguez-Pérez et $a{ }^{17}{ }^{17}$ reported that phenolics including dihydroferulic acid glucuronide, procyanidin A dimer, quercetin glucoside, myricetin and prodelphinidin B decreased the surface hydrophobicity of UPEC in vitro, resulting in potential reductions in adhesive forces. By using the atomic force microscopy technique, Gupta and colleagues ${ }^{16}$ found that a crude cranberry extract as well as flavonols including quercetin-3-O-galactoside and myricetin-3-O-galactoside showed anti-adhesion properties on both non-pathogenic and antibiotic resistant $E$. coli strains. In another study performed by Rafsanjany et al. ${ }^{25}$ adhesion of E. coli strain NU14, which expresses type 1 fimbriae, was reduced by both the cranberry extract and the PAC-free cranberry extract, which is consistent with previous studies demonstrating that PACs inhibit P-type E. coli.

Although it has been reported that certain cranberry parent compounds are bioavailable, the concentration in urine is rather low. ${ }^{46}$ Recent studies tested gut microbial-derived cranberry metabolites and their potential bacteria anti-adhesion activity. Two of the most abundant metabolites produced following cranberry consumption, hippuric acid and $\alpha$-hippuric acid, exhibited in vitro anti-adhesion effects on UPEC at physiologically relevant concentrations (100, 250 and $500 \mu \mathrm{M})$. In addition, catechol, benzoic acid, vanillic acid, phenylacetic acid and 3,4-dihydroxyphenylacetic acid were able to inhibit the adherence of UPEC to uroepithelia cells in a concentrationdependent manner from $100-500 \mu \mathrm{M},{ }^{18}$ although further studies are needed. One of the cranberry PAC degradation products, valerolactone and its derivatives, showed anti-adhesion properties at a concentration of $100 \mu \mathrm{M}$, indicating their potential biologically relevant activity in urine. ${ }^{19}$

Despite encouraging observation from cell-based studies, few studies have investigated the ex vivo urinary anti-adhesion effect against type $1 \mathrm{E}$. coli, combining both a human intervention component and an in vitro component to study exposure to the cranberry product occurring at expected intake levels in humans and the presence of anti-adhesive cranberry components in the urine. Di Martino et $a .^{24}$ found that urine from cranberry juice consumers inhibited the adhesion of $E$. coli with type 1 fimbriae. In another study by Rafsanjany et al., ${ }^{25}$ subjects consumed $600 \mathrm{mg}$ cranberry extracts for either 3 or 7 days. Urine collected from those participants had a significantly enhanced anti-adhesion effect on UPEC strain NU14, a clinical cystitis isolate, indicating a beneficial effect of cranberry consumption on type $1 \mathrm{E}$. coli-associated UTIs.

The current study demonstrates ex vivo urinary antiadhesion activity against both P-type and type $1 \mathrm{E}$. coli, following consumption of cranberry formulated in a chew product. Interestingly, a sustained anti-adhesion benefit was achieved for up to 24 hours when two cranberry chews were consumed daily, one in the morning and one in the evening. Our MRHA results demonstrating that consumption of the cranberry chew supplement helped to prevent P-type E. coli from adhering to cell receptors are consistent with other reports using cranberry juices, dried cranberries, powders, and capsules as test products. $^{9,13,21,47,48}$

\section{Conclusions}

In conclusion, our study demonstrated that human urine following consumption of cranberry thealth ${ }^{\mathrm{TM}}$ cranberry supplement (cranberry chew) had the ability to inhibit the adherence of both $\mathrm{P}$ type and type 1 uropathogenic $E$. coli. Results utilizing assays specific for each bacterial type indicate that consuming a second dose of cranberry chew 12 hours after the initial dose provided extended protection from bacterial adhesion for up to 24 hours. Further human trials are needed to correlate the level of ex vivo anti-adhesion activity with prevention of clinical UTIs.

\section{Conflicts of interest}

Haiyan Liu, Derek J. Zhang and Christina Khoo are employees of Ocean Spray. Dr Amy B. Howell is an independent university researcher and collaborator with no conflict of interest to declare. 


\section{Acknowledgements}

This study was funded by Ocean Spray Cranberries, Inc.

\section{References}

1 G. B. D. Disease, I. Injury and C. Prevalence, Global, regional, and national incidence, prevalence, and years lived with disability for 328 diseases and injuries for 195 countries, 1990-2016: a systematic analysis for the Global Burden of Disease Study 2016, Lancet, 2017, 390, 1211-1259.

2 D. G. Moriel and M. A. Schembri, Vaccination approaches for the prevention of urinary tract infection, Curr. Pharm. Biotechnol., 2013, 14, 967-974.

3 B. Foxman, The epidemiology of urinary tract infection, Nat. Rev. Urol., 2010, 7, 653-660.

4 Z. Fu, D. Liska, D. Talan and M. Chung, Cranberry Reduces the Risk of Urinary Tract Infection Recurrence in Otherwise Healthy Women: A Systematic Review and Meta-Analysis, J. Nutr., 2017, 147, 2282-2288.

5 A. Luis, F. Domingues and L. Pereira, Can Cranberries Contribute to Reduce the Incidence of Urinary Tract Infections? A Systematic Review with Meta-Analysis and Trial Sequential Analysis of Clinical Trials, J. Urol., 2017, 198, 614-621.

6 K. C. Maki, K. L. Kaspar, C. Khoo, L. H. Derrig, A. L. Schild and K. Gupta, Consumption of a cranberry juice beverage lowered the number of clinical urinary tract infection episodes in women with a recent history of urinary tract infection, Am. J. Clin. Nutr., 2016, 103, 1434-1442.

7 R. G. Jepson, G. Williams and J. C. Craig, Cranberries for preventing urinary tract infections, Cochrane Database Syst. Rev., 2012, 10, CD001321.

8 T. M. Hooton, Clinical practice. Uncomplicated urinary tract infection, N. Engl. J. Med., 2012, 366, 1028-1037.

9 C. Khoo and H. Liu, in Polyphenols: Prevention and treatment of human disease, Academic Press, Cambridge, Massachusetts, United States, 2nd edn, 2018, ch. 8, pp. 89-105, DOI: 10.1016/B978-0-12-813008-7.00008-4.

10 B. Xie, G. Zhou, S. Y. Chan, E. Shapiro, X. P. Kong, X. R. Wu, T. T. Sun and C. E. Costello, Distinct glycan structures of uroplakins Ia and Ib: structural basis for the selective binding of FimH adhesin to uroplakin Ia, J. Biol. Chem., 2006, 281, 14644-14653.

11 G. Kallenius, S. Svenson, R. Mollby, B. Cedergren, H. Hultberg and J. Winberg, Structure of carbohydrate part of receptor on human uroepithelial cells for pyelonephritogenic Escherichia coli, Lancet, 1981, 2, 604-606.

12 M. S. Conover, S. Ruer, J. Taganna, V. Kalas, H. De Greve, J. S. Pinkner, K. W. Dodson, H. Remaut and S. J. Hultgren, Inflammation-Induced Adhesin-Receptor Interaction Provides a Fitness Advantage to Uropathogenic E. coli during Chronic Infection, Cell Host Microbe, 2016, 20, 482-492.

13 A. B. Howell, J. D. Reed, C. G. Krueger, R. Winterbottom, D. G. Cunningham and M. Leahy, A-type cranberry proanthocyanidins and uropathogenic bacterial antiadhesion activity, Phytochemistry, 2005, 66, 2281-2291.

14 B. D. Mathison, L. L. Kimble, K. L. Kaspar, C. Khoo and B. P. Chew, Development and validation of a sensitive, highthroughput bioassay for the adhesion of radiolabeled E. coli to uroepithelial cells in vitro, J. Nat. Prod., 2013, 76, 1605-1611.

15 L. L. Kimble, B. D. Mathison, K. L. Kaspar, C. Khoo and B. P. Chew, Development of a fluorometric microplate antiadhesion assay using uropathogenic Escherichia coli and human uroepithelial cells, J. Nat. Prod., 2014, 77, 1102-1110.

16 P. Gupta, B. Song, C. Neto and T. A. Camesano, Atomic force microscopy-guided fractionation reveals the influence of cranberry phytochemicals on adhesion of Escherichia coli, Food Funct., 2016, 7, 2655-2666.

17 C. Rodríguez-Pérez, R. Quirantes-Pine, J. Uberos, C. Jimenez-Sanchez, A. Pena and A. Segura-Carretero, Antibacterial activity of isolated phenolic compounds from cranberry (Vaccinium macrocarpon) against Escherichia coli, Food Funct., 2016, 7, 1564-1573.

18 D. G. de Llano, A. Esteban-Fernandez, F. Sanchez-Patan, P. J. Martinlvarez, M. V. Moreno-Arribas and B. Bartolome, Anti-Adhesive Activity of Cranberry Phenolic Compounds and Their Microbial-Derived Metabolites against Uropathogenic Escherichia coli in Bladder Epithelial Cell Cultures, Int. J. Mol. Sci., 2015, 16, 12119-12130.

19 P. Mena, D. G. de Llano, N. Brindani, A. EstebanFernandez, C. Curti, M. V. Moreno-Arribas, D. D. Rio and B. Bartolome, 5-(3',4'-Dihydroxyphenyl)- $\gamma$-valerolactone and its sulphate conjugates, representative circulating metabolites of flavan-3-ols, exhibit anti-adhesive activity against uropathogenic Escherichia coli in bladder epithelial cells, J. Funct. Foods, 2017, 29, 275-280.

20 K. Gupta, M. Y. Chou, A. Howell, C. Wobbe, R. Grady and A. E. Stapleton, Cranberry products inhibit adherence of p-fimbriated Escherichia coli to primary cultured bladder and vaginal epithelial cells, J. Urol., 2007, 177, 2357-2360.

21 A. B. Howell, H. Botto, C. Combescure, A. B. Blanc-Potard, L. Gausa, T. Matsumoto, P. Tenke, A. Sotto and J. P. Lavigne, Dosage effect on uropathogenic Escherichia coli anti-adhesion activity in urine following consumption of cranberry powder standardized for proanthocyanidin content: a multicentric randomized double blind study, BMC Infect. Dis., 2010, 10, 94.

22 K. L. Kaspar, A. B. Howell and C. Khoo, A randomized, double-blind, placebo-controlled trial to assess the bacterial anti-adhesion effects of cranberry extract beverages, Food Funct., 2015, 6, 1212-1217.

23 B. D. Mathison, L. L. Kimble, K. L. Kaspar, C. Khoo and B. P. Chew, Consumption of cranberry beverage improved endogenous antioxidant status and protected against bacteria adhesion in healthy humans: a randomized controlled trial, Nutr. Res., 2014, 34, 420-427.

24 P. Di Martino, R. Agniel, K. David, C. Templer, J. L. Gaillard, P. Denys and H. Botto, Reduction of Escherichia coli adherence to uroepithelial bladder cells after consumption of cranberry juice: a double-blind ran- 
domized placebo-controlled cross-over trial, World J. Urol., 2006, 24, 21-27.

25 N. Rafsanjany, J. Senker, S. Brandt, U. Dobrindt and A. Hensel, In Vivo Consumption of Cranberry Exerts ex Vivo Antiadhesive Activity against FimH-Dominated Uropathogenic Escherichia coli: A Combined in Vivo, ex Vivo, and in Vitro Study of an Extract from Vaccinium macrocarpon, J. Agric. Food Chem., 2015, 63, 8804-8818.

26 S. L. Chen, C. S. Hung, J. Xu, C. S. Reigstad, V. Magrini, A. Sabo, D. Blasiar, T. Bieri, R. R. Meyer, P. Ozersky, J. R. Armstrong, R. S. Fulton, J. P. Latreille, J. Spieth, T. M. Hooton, E. R. Mardis, S. J. Hultgren and J. I. Gordon, Identification of genes subject to positive selection in uropathogenic strains of Escherichia coli: a comparative genomics approach, Proc. Natl. Acad. Sci. U. S. A., 2006, 103, 5977-5982.

27 K. M. Lee, J. Lim, S. Nam, M. Y. Yoon, Y. K. Kwon, B. Y. Jung, Y. Park, S. Park and S. S. Yoon, Inhibitory effects of broccoli extract on Escherichia coli O157:H7 quorum sensing and in vivo virulence, FEMS Microbiol. Lett., 2011, 321, 67-74.

28 R. Puupponen-Pimia, L. Nohynek, C. Meier, M. Kahkonen, M. Heinonen, A. Hopia and K. M. Oksman-Caldentey, Antimicrobial properties of phenolic compounds from berries, J. Appl. Microbiol., 2001, 90, 494-507.

29 M. A. Martin, S. Ramos, R. Mateos, J. P. Marais, L. BravoClemente, C. Khoo and L. Goya, Chemical characterization and chemo-protective activity of cranberry phenolic powders in a model cell culture. Response of the antioxidant defenses and regulation of signaling pathways, Food Res. Int., 2015, 71, 68-82.

30 R. L. Prior, E. Fan, H. Ji, A. Howell, C. Nio, M. J. Payne and J. Reed, Multi-laboratory validation of a standard method for quantifying proanthocyanidins in cranberry powders, J. Sci. Food Agric., 2010, 90, 1473-1478.

31 R. J. Robbins, J. Leonczak, J. Li, J. C. Johnson, T. Collins, C. Kwik-Uribe and H. H. Schmitz, Determination of flavanol and procyanidin (by degree of polymerization 1-10) content of chocolate, cocoa liquors, powder(s), and cocoa flavanol extracts by normal phase high-performance liquid chromatography: collaborative study, J. AOAC Int., 2012, 95, 1153-1160.

32 J. C. Sanchez-Rangel, J. Benavides, J. B. Heredia, L. Cisneros-Vevallos and D. A. Jacobo-Velazquez, The Folin-Ciocalteu assay revisited: improvement of its specificity for total phenolic content determination, Anal. Methods, 2013, 5, 5990-5999.

33 P. N. Brown and P. R. Shipley, Determination of anthocyanins in cranberry fruit and cranberry fruit products by high-performance liquid chromatography with ultraviolet detection: single-laboratory validation, J. AOAC Int., 2011, 94, 459-466.

34 J. L. Duncan, Differential effect of Tamm-Horsfall protein on adherence of Escherichia coli to transitional epithelial cells, J. Infect. Dis., 1988, 158, 1379-1382.

35 F. Serafini-Cessi, N. Malagolini and D. Cavallone, TammHorsfall glycoprotein: biology and clinical relevance, Am. J. Kidney Dis., 2003, 42, 658-676.
36 R. P. Feliciano, C. G. Krueger, D. Shanmuganayagam, M. M. Vestling and J. D. Reed, Deconvolution of matrixassisted laser desorption/ionization time-of-flight mass spectrometry isotope patterns to determine ratios of A-type to B-type interflavan bonds in cranberry proanthocyanidins, Food Chem., 2012, 135, 1485-1493.

37 R. P. Feliciano, J. J. Meudt, D. Shanmuganayagam, C. G. Krueger and J. D. Reed, Ratio of "A-type" to "B-type" proanthocyanidin interflavan bonds affects extra-intestinal pathogenic Escherichia coli invasion of gut epithelial cells, J. Agric. Food Chem., 2014, 62, 3919-3925.

38 K. Ou, S. S. Percival, T. Zou, C. Khoo and L. Gu, Transport of cranberry A-type procyanidin dimers, trimers, and tetramers across monolayers of human intestinal epithelial Caco-2 cells, J. Agric. Food Chem., 2012, 60, 1390-1396.

39 K. Ou, P. Sarnoski, K. R. Schneider, K. Song, C. Khoo and L. Gu, Microbial catabolism of procyanidins by human gut microbiota, Mol. Nutr. Food Res., 2014, 58, 2196-2205.

40 H. E. Khoo, A. Azlan, S. T. Tang and S. M. Lim, Anthocyanidins and anthocyanins: colored pigments as food, pharmaceutical ingredients, and the potential health benefits, Food Nutr. Res., 2017, 61, 1361779.

41 D. G. Cunningham, S. A. Vannozzi, R. Turk, R. Roderick, E. O'Shea and K. Brilliant, in Nutraceutical Beverages, chemistry, nutrition and health benefits, ACS Publications, Washington D.C., 2003, ch. 4, pp. 35-51, DOI: 10.1021/bk2004-0871.ch004.

42 I. O. Vvedenskaya, R. T. Rosen, J. E. Guido, D. J. Russell, K. A. Mills and N. Vorsa, Characterization of flavonols in cranberry (Vaccinium macrocarpon) powder, J. Agric. Food Chem., 2004, 52, 188-195.

$43 \mathrm{~W}$. Zheng and S. Y. Wang, Oxygen radical absorbing capacity of phenolics in blueberries, cranberries, chokeberries, and lingonberries, J. Agric. Food Chem., 2003, 51, 502509.

44 S. A. Aherne and N. M. O'Brien, Dietary flavonols: chemistry, food content, and metabolism, Nutrition, 2002, 18, 75-81.

45 W. Mullen, S. C. Marks and A. Crozier, Evaluation of phenolic compounds in commercial fruit juices and fruit drinks, J. Agric. Food Chem., 2007, 55, 3148-3157.

46 D. L. McKay, C. Y. Chen, C. A. Zampariello and J. B. Blumberg, Flavonoids and phenolic acids from cranberry juice are bioavailable and bioactive in healthy older adults, Food Chem., 2015, 168, 233-240.

47 J. A. Greenberg, S. J. Newmann and A. B. Howell, Consumption of sweetened dried cranberries versus unsweetened raisins for inhibition of uropathogenic Escherichia coli adhesion in human urine: a pilot study, J. Altern. Complement. Med., 2005, 11, 875-878.

48 A. Howell, D. Souza, M. Roller and E. Fromentin, Comparison of the Anti-Adhesion Activity of Three Different Cranberry Extracts on Uropathogenic P-fimbriated Escherichia coli: a Randomized, Doubleblind, Placebo Controlled, Ex Vivo, Acute Study, Nat. Prod. Commun., 2015, 10, 1215-1218. 